\title{
Admission During Off-Hours Does Not Affect Long-Term Clinical Outcomes of Japanese Patients with Acute Myocardial Infarction J-MINUET Substudy
}

\author{
Satoru Suwa, ${ }^{1}$ MD, Manabu Ogita, ${ }^{1}$ MD, Hideki Ebina, ${ }^{1}$ MD, Koichi Nakao, ${ }^{2}$ MD, Yukio Ozaki, ${ }^{3}$ MD, \\ Kazuo Kimura, ${ }^{4}$ MD, Junya Ako, ${ }^{5}$ MD, Teruo Noguchi, ${ }^{6}$ MD, Satoshi Yasuda,${ }^{6}$ MD, \\ Kazuteru Fujimoto, ${ }^{7}$ MD, Yasuharu Nakama, ${ }^{8}$ MD, Takashi Morita, ${ }^{9}$ MD, Wataru Shimizu, ${ }^{10}$ MD, \\ Yoshihiko Saito, ${ }^{11} \mathrm{MD}$, Atsushi Hirohata, ${ }^{12} \mathrm{MD}$, Yasuhiro Morita, ${ }^{13} \mathrm{MD}$, Teruo Inoue ${ }^{14} \mathrm{MD}$, \\ Atsunori Okamura, ${ }^{15} \mathrm{MD}$, Toshiaki Mano, ${ }^{16} \mathrm{MD}$, Kazuhito Hirata, ${ }^{17} \mathrm{MD}$, Kengo Tanabe,${ }^{18} \mathrm{MD}$, \\ Yoshisato Shibata, ${ }^{19} \mathrm{MD}$, Mafumi Owa, ${ }^{20} \mathrm{MD}$, Kenichi Tsujita, ${ }^{21} \mathrm{MD}$, Hiroshi Funayama, ${ }^{22} \mathrm{MD}$, \\ Nobuaki Kokubu, ${ }^{23}$ MD, Ken Kozuma ${ }^{24}$ MD, Shiro Uemura, ${ }^{25}$ MD, Tetsuya Toubaru, ${ }^{26}$ MD, \\ Keijiro Saku, ${ }^{27} \mathrm{MD}$, Shigeru Oshima, ${ }^{28} \mathrm{MD}$, Kunihiro Nishimura, ${ }^{29} \mathrm{MD}$, \\ Yoshihiro Miyamoto, ${ }^{29} \mathrm{MD}$, Hisao Ogawa, ${ }^{29} \mathrm{MD}$ and Masaharu Ishihara, ${ }^{30} \mathrm{MD}$ \\ on behalf of J-MINUET investigators
}

\begin{abstract}
Summary
Discordant results have been reported on outcomes of acute myocardial infarction (AMI) patients who present during off-hours.

We investigated 3283 consecutive patients with AMI who were selected from the prospective, nationwide, multicenter registry (J-MINUET) database comprising 28 institutions in Japan between July 2012 and March 2014 to determine the current impact of off-hours presentation (defined as weekends, holidays, and weekdays from 8:01 PM to 7:59 AM) at hospitals on long-term clinical outcomes. The primary endpoint was a composite of all-cause death, non-fatal MI, non-fatal stroke, cardiac failure, and urgent revascularization for unstable angina for up to 3 years from the index event.

During off-hours, $52 \%$ of patients presented. Primary percutaneous coronary intervention was performed in $85 \%$ of patients, and the door-to-balloon time was comparable between off-hours and regular hours (74, interquartile range [IQR] 52 to 113 versus 75 , IQR 52 to 126 minutes, $P=0.34$ ). Rate of overall primary endpoint overall did not overall significantly differ $(25.3 \%$ versus $23.5 \%$, log-rank $P=0.26$ ), in patients with STelevation myocardial infarction (STEMI) (log-rank $P=0.93$ ) and in patients with non-ST-elevation myocardial infarction (NSTEMI) (log-rank $P=0.14$ ). Multivariate Cox regression analysis showed that off-hours presentation was not significantly associated with long-term clinical events in all cohorts.
\end{abstract}

From the ${ }^{1}$ Department of Cardiology, Juntendo University Shizuoka Hospital, Izunokuni, Japan, ${ }^{2}$ Division of Cardiology, Saiseikai Kumamoto Hospital Cardiovascular Center, Kumamoto, Japan, ${ }^{3}$ Department of Cardiology, Fujita Health University Hospital, Toyoake, Japan, ${ }^{4}$ Division of Cardiology, Yokohama City University Medical Center, Yokohama, Japan, ${ }^{5}$ Department of Cardiovascular Medicine, Kitasato University, Sagamihara, Japan, ${ }^{6}$ Department of Cardiovascular Medicine, National Cerebral and Cardiovascular Center, Suita, Japan, ${ }^{7}$ Department of Cardiology, National Hospital Organization Kumamoto Medical Center, Kumamoto, Japan, ${ }^{8}$ Department of Cardiology, Hiroshima City Hospital, Hiroshima, Japan, ${ }^{9}$ Division of Cardiology, Osaka General Medical Center, Osaka, Japan, ${ }^{10}$ Department of Cardiovascular Medicine, Nippon Medical School, Tokyo, Japan, ${ }^{11}$ First Department of Internal Medicine, Nara Medical University, Kashihara, Japan, ${ }^{12}$ Department of Cardiovascular Medicine, The Sakakibara Heart Institute of Okayama, Okayama, Japan, ${ }^{13}$ Department of Cardiology, Ogaki Municipal Hospital, Ogaki, Japan, ${ }^{14}$ Department of Cardiovascular Medicine, Dokkyo Medical University, Tochigi, Japan, ${ }^{15}$ Department of Cardiology, Sakurabashi Watanabe Hospital, Osaka, Japan, ${ }^{16}$ Cardiovascular Center, Kansai Rosai Hospital, Amagasaki, Japan, ${ }^{17}$ Department of Cardiology, Okinawa Prefectural Chubu Hospital, Uruma, Japan, ${ }^{18}$ Division of Cardiology, Mitsui Memorial Hospital, Tokyo, Japan, ${ }^{19}$ Department of Cardiology, Miyazaki Medical Association Hospital, Miyazaki, Japan, ${ }^{20}$ Department of Cardiovascular Medicine, Suwa Red Cross Hospital, Suwa, Japan, ${ }^{21}$ Department of Cardiovascular Medicine, Graduate School of Medical Sciences, Kumamoto University, Kumamoto, Japan, ${ }^{22}$ Department of Integrated Medicine, Saitama Medical Center Jichi Medical University, Saitama, Japan, ${ }^{23}$ Department of Cardiovascular, Renal and Metabolic Medicine, Sapporo Medical University, Sapporo, Japan, ${ }^{24}$ Department of Cardiology, Teikyo University, Tokyo, Japan, ${ }^{25}$ Department of Cardiology, Kawasaki Medical School, Kurashiki, Japan, ${ }^{26}$ Department of Cardiology, Sakakibara Heart Institute, Tokyo, Japan, ${ }^{27}$ Department of Cardiology, Fukuoka University School of Medicine, Fukuoka, Japan, ${ }^{28}$ Department of Cardiology, Gunma Prefectural Cardiovascular Center, Maebashi, Japan, ${ }^{29}$ Department of Preventive Medicine and Epidemiologic Informatics, National Cerebral and Cardiovascular Center, Suita, Japan and ${ }^{30}$ Division of Coronary Artery Disease, Hyogo College of Medicine, Nishinomiya, Japan.

This study was supported by the Intramural Research Fund (Grant number 23-4-5) for Cardiovascular Diseases at the National Cerebral and Cardiovascular Center.

Address for correspondence: Manabu Ogita, MD, Department of Cardiology, Juntendo University Shizuoka Hospital, 1129 Nagaoka, Izunokuni, Shizuoka 410-2295, Japan. E-mail: m-ogita@sa2.so-net.ne.jp

Received for publication August 20, 2019. Revised and accepted November 11, 2019.

Released in advance online on J-STAGE March 14, 2020.

doi: 10.1536/ihj.19-434

All rights reserved by the International Heart Journal Association. 
The impact of presentation during off-hours or regular hours on the long-term clinical outcomes of Japanese patients with AMI is comparable in contemporary practice.

(Int Heart J 2020; 61: 215-222)

Key words: Coronary artery disease, Off-hour presentation, Outcome measurements

A cute myocardial infarction (AMI) remains a leading cause of mortality and morbidity worldwide. ${ }^{1)}$ Percutaneous coronary intervention (PCI) is an established revascularization therapy for patients with $\mathrm{AMI}^{2)}$ but still challenging in the high-risk AMI population. ${ }^{3,4)}$ Mortality rates are reportedly higher among patients with AMI who are admitted during off-hours (nights, weekends, and holidays) than those admitted during regular hours. ${ }^{5-8)}$ In contrast, an equal number of conflicting results have been reported, ${ }^{9-12)}$ which raises the question as to whether the quality of care and clinical outcomes differ depending on the time of admission to hospital. ${ }^{13,14)}$ We previously found no significant differences in short-term (in-hospital) clinical outcomes between regular hours and off-hours. ${ }^{15)}$ In this study, most patients $(85 \%)$ were treated with PCI. Various factors during off-hours such as availability of cardiologists, support staff for the cardiac catheterization, the number of skilled nursing staff, and human factors such as sleep deprivation and fatigue may attribute to the PCI-related late events. Thereafter, we conducted a post hoc analysis of the J-MINUET study to evaluate associations between off-hours presentation and long-term clinical outcomes in patients with AMI in contemporary practice in Japan.

\section{Methods}

Study participants: The multicenter, prospective, observational J-MINUET (Japan Registry of Acute Myocardial Infarction Diagnosed by Universal Definition) study enrolled consecutive patients with AMI who presented at 28 Japanese medical institutions between July 2012 and March 2014. ${ }^{16)}$ The diagnosis of AMI was based on the ESC/ACC Foundation (ACCF)/American Heart Association (AHA)/World Heart Federation Task Force for the Universal Definition Myocardial infarction. ${ }^{17)}$ Only type 1 (spontaneous MI related to ischemia due to primary coronary events) and type 2 (MI secondary to ischemia due to either increased oxygen demand or decreased supply) were included in this study. The baseline demographic and clinical characteristics of 3283 patients with AMI were evaluated. ST-elevation myocardial infarction (STEMI) was diagnosed in the presence of new ST elevation at the $\mathrm{J}$ point in at least two contiguous leads. New or presumably new left bundle branch block was considered equivalent to STEMI. This study proceeded according to the Declaration of Helsinki with the approval of the ethics committees of every participating institution.

Outcome measurements: The primary outcome was a composite of all-cause death, non-fatal MI, non-fatal stroke, cardiac failure, and urgent revascularization of unstable angina after the index MI for up to 3 years among patients presenting either during regular hours (defined as weekdays from 8:00 AM to 8:00 PM) or off-hours (defined as weekends, holidays, and weekdays from 8:01 PM to $7: 59 \mathrm{AM}){ }^{18)}$ In brief, we included only types 1 and 2 non-fatal MI and defined cardiac failure as heart failure requiring hospital admission. Secondary endpoints defined (1) death; (2) composite of death and non-fatal MI; (3) death, non-fatal MI, and non-fatal stroke; and (4) composite of death, non-fatal MI, non-fatal stroke, and cardiac failure.

All patients were clinically followed up after the index MI through reviews of medical records, telephone contact, and postal-mailed questionnaires for up to 3 years.

Statistical analysis: We used information derived from the database of the J-MINUET study for this sub-analysis. Continuous variables presented as medians (25th to 75 th percentiles) were compared using unpaired $t$-tests or Mann-Whitney $U$-tests. Categorical variables are expressed as numbers and ratios (\%) and were compared using chi-square or Fisher's exact probability tests. Unadjusted cumulative event rates were estimated using Kaplan-Meier curves and compared between groups using log-rank tests overall and in STEMI and NSTEMI cohorts. Hazard ratios (HR) and 95\% confidence intervals (CI) were determined using Cox proportional hazards models. Determinants of primary outcome were assessed using univariate and multivariate Cox regression analyses. Multivariable analyses included the established prognostic risk factors for cardiac events according to primary paper (adjusted for age, sex, hypertension, diabetes, dyslipidemia, chronic kidney disease, current smoking, previous MI, previous stroke, Killip class, type of MI, logarithm of time from onset to admission, urgent coronary angiography, primary PCI, and urgent bypass surgery). ${ }^{16)}$ Values with $P<0.05$ were considered statistically significant. All data were statistically analyzed using JMP12.2 (SAS Institute Inc., Cary, NC, USA).

\section{Results}

Baseline characteristics: Among the 3283 AMI patients in the present substudy, $52 \%$ of them were presented during off-hours (Figure 1). The baseline clinical characteristics of the patients are shown in Table I. The median age was 69 (IQR 61-78). The patients who presented during off-hours were younger and had a higher incidence of STEMI and advanced Killip class than those who presented during regular hours. The median amount of time that elapsed between symptom onset and presentation time was shorter for patients who presented during off-hours than regular hours (120, IQR 60-256 versus 215, IQR 90610 minutes, $P<0.0001)$. Primary PCI was performed in $85 \%$ of the patients. Door-to-balloon time was comparable 


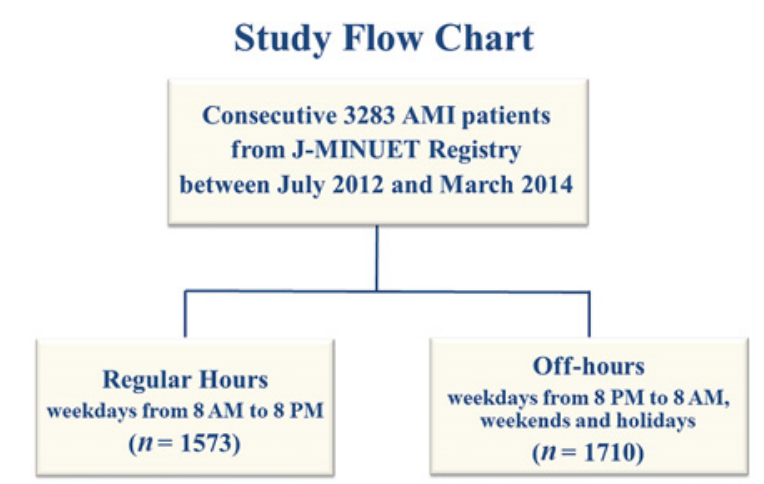

Primary endpoint; all-cause death, non-fatal MI, non-fatal stroke, cardiac failure and urgent revascularization for unstable angina up to 3 years

Figure 1. Study flow chart.

between the groups (74, IQR 52-113 versus 75, IQR 52126 minutes, $P=0.34$; Figure 2). Door-to-balloon time was also comparable between patients with STEMI who presented during off-hours and regular hours (64, IQR 4892 versus 67, IQR 50-93 minutes, $P=0.34$; Figure 3).

Clinical outcomes: Rates of major cardiovascular events during 3 years of follow up were comparable overall and in STEMI and NSTEMI $(25.3 \%$ versus $23.5 \%$, log-rank $P$ $=0.26 ; 23.0 \%$ versus $21.2 \%, \log$-rank $P=0.93$ and $31.4 \%$ versus $27.7 \%$, log-rank $P=0.14$, respectively; Figure 4). Kaplan-Meier curves of secondary endpoints are shown in Figure 5. In multivariate Cox regression analysis, off-hour presentation was not significantly associated with the long-term adverse clinical outcomes overall and in STEMI and NSTEMI cohorts (HR, 1.03, 95\% CI 0.87$1.21, P=0.26$; HR $1.01,95 \%$ CI $0.82-1.23, P=0.93$; HR $1.10,95 \%$ CI $0.84-1.44, P=0.47$, respectively; Table II).

\section{Discussion}

We analyzed 3283 patients with AMI from the prospective, nationwide, multicenter registry in Japan (JMINUET). We found that long-term prognosis was comparable for both patients with AMI who presented during both regular hours and off-hours and that presenting during off-hours was not significantly associated with longterm outcomes after adjusting for risk factors.

Previous studies have shown that short-term mortality rates among patients with AMI are worse if they present to hospitals during off-hours compared with regular hours. ${ }^{5-8)}$ Several proposed explanations include a lower likelihood of receiving evidence-based treatment or timely reperfusion therapies, ${ }^{19)}$ variations in healthcare delivery between daytime and nighttime, fewer hospital staff during nighttime, and staff level of expertise. ${ }^{20,21)}$ In contrast, a few number of studies have found an association between off-hours presentation and outcomes: long-term adverse clinical outcomes were worse among patient admitted during off-hours compared with during regular hours. ${ }^{22,23)}$ In contrast, recent studies have found no differences in long-term outcomes of patients with AMI regardless of the time of presentation and our results are com- patible with these studies. ${ }^{24-26)}$ A recent report from a highvolume center in the Netherlands found that the clinical outcomes of 4352 consecutive STEMI patients between 2000 and 2009 were similar for up to 4 years after the index primary PCI. ${ }^{24)}$ The Swedish Coronary Angiograpy and Angioplasty Registry study of 7136 patients with STEMI who underwent primary PCI between 2004 and 2013 found similar long-term prognoses during regularhours and off-hours presentation. ${ }^{25}$ Cubeddu et al. reported similar short- and long-term clinical outcomes for patients in the prospective cohort database of the A Harmonizing Outcomes With RevasculariZatiON and Stents in AMI (HORIZONS-AMI) study. ${ }^{26)}$ Our findings are consistent with these recent results. The comparable outcomes between the regular-hours and off-hours admissions can be explained by overall improvements in STEMI programs and better-quality performance regardless of the time of presentation.

Previous studies have identified delays in door-toballoon time during off-hours ${ }^{27,28)}$ and a meta-analysis found longer door-to-balloon time and worse clinical outcomes for patients who presented during off-hours than during regular hours. ${ }^{8)}$ A recent prospective multicenter cohort study showed an almost linear relationship between contact-to-balloon times and mortality in patients with STEMI treated within 60-180 minutes of the first medical contact. ${ }^{29)}$ Dasari et al. described an average delay in door-to-balloon time of 16 minutes when patients present off-hours. ${ }^{30)}$ We found here that the door-to-balloon time among patients requiring primary PCI was comparable between regular-hours and off-hours presentation, indicating that primary PCI facilities in Japan offer a consistent quality of healthcare, and this might be a factor in the favorable clinical outcomes of patients who present during offhours.

In contrast, total ischemic time has not improved recently and this correlates with increased risk of adverse outcomes. ${ }^{31)}$ A report from CREDO Kyoto registry in Japan showed that an onset-to-balloon time less than 3 hours provides better 3 year clinical outcomes, ${ }^{32)}$ suggesting that continuous effort to reduce total ischemic time is required to improve outcomes for patients with AMI. This study identified shorter transportation time in patients presented during off-hours. Several possible explanations for the inconsistent results concerning the duration of transportation have been suggested. One is that Japan has a unique healthcare system in which social health insurance offers universal healthcare unlike many other developed countries. ${ }^{33)}$ This system allows patients to call emergency medical services at any time and be taken to a hospital at no change. Consequently, most patients with AMI in Japan are managed at centers with $24 / 7$ primary PCI facilities. Another factor is that significantly more frequent interfacility transfer during regular hours results in longer transportation time. A report from the CREDO KYOTO registry in Japan found that interfacility transfer was associated with worse clinical outcomes for patients with STEMI undergoing primary PCI. ${ }^{34)}$ The higher rate of interfacility transfer during regular hours might not result in an outcome difference between regular hours and offhours. Increased focus on prehospital management and a 
Table I. Baseline Clinical Characteristics of the Study Population

\begin{tabular}{|c|c|c|c|}
\hline & Regular-hours $(n=1573)$ & Off-hours $(n=1710)$ & $P$ value \\
\hline Age, years & $71(62-79)$ & $68(59-78)$ & $<0.0001$ \\
\hline Male & $74.4 \%$ & $76.0 \%$ & 0.31 \\
\hline Hypertension & $68.7 \%$ & $64.5 \%$ & 0.0115 \\
\hline Diabetes & $36.7 \%$ & $36.0 \%$ & 0.68 \\
\hline Dyslipidemia & $52.0 \%$ & $51.9 \%$ & 0.94 \\
\hline Current smoking & $31.8 \%$ & $36.0 \%$ & 0.0133 \\
\hline CKD & $44.6 \%$ & $44.9 \%$ & 0.84 \\
\hline Prior MI & $12.2 \%$ & $12.0 \%$ & 0.83 \\
\hline Prior PCI & $15.7 \%$ & $14.9 \%$ & 0.55 \\
\hline Prior CABG & $2.7 \%$ & $3.1 \%$ & 0.52 \\
\hline Prior stroke & $12.2 \%$ & $10.5 \%$ & 0.12 \\
\hline Diagnosis & & & $<0.0001$ \\
\hline STEMI & $64.8 \%$ & $72.6 \%$ & \\
\hline NSTEMI & $35.2 \%$ & $27.4 \%$ & \\
\hline Killip Classification & & & 0.0079 \\
\hline Class 1 & $77.9 \%$ & $73.5 \%$ & \\
\hline Class 2 & $9.0 \%$ & $9.5 \%$ & \\
\hline Class 3 & $4.2 \%$ & $6.5 \%$ & \\
\hline Class 4 & $8.9 \%$ & $10.5 \%$ & \\
\hline Transferred by & & & $<0.0001$ \\
\hline Emergency transfer & $44.8 \%$ & $58.2 \%$ & \\
\hline Walk-in & $20.2 \%$ & $16.0 \%$ & \\
\hline In-hospital & $3.7 \%$ & $2.6 \%$ & \\
\hline From other hospital & $30.0 \%$ & $19.2 \%$ & \\
\hline Unknown & $4.3 \%$ & $4.0 \%$ & \\
\hline \multicolumn{4}{|l|}{ Onset to presentation time, minutes } \\
\hline Overall & $215(90-610)$ & $120(60-256)$ & $<0.0001$ \\
\hline NSTEMI & $278(95-780)$ & $135(63-309)$ & $<0.0001$ \\
\hline STEMI & $185(85-503)$ & $119(60-239)$ & $<0.0001$ \\
\hline Urgent CAG & $92.7 \%$ & $93.5 \%$ & 0.42 \\
\hline Urgent Revascularization & & & 0.07 \\
\hline PCI & $83.9 \%$ & $86.2 \%$ & \\
\hline $\mathrm{CABG}$ & $2.6 \%$ & $1.6 \%$ & \\
\hline None & $13.6 \%$ & $12.2 \%$ & \\
\hline Target lesion & & & 0.32 \\
\hline LAD as IRA & $45.5 \%$ & $43.6 \%$ & \\
\hline Multivessel disease & $44.0 \%$ & $43.3 \%$ & 0.72 \\
\hline Initial TIMI flow 0/1 & $56.8 \%$ & $63.8 \%$ & $<0.0001$ \\
\hline IABP use & $15.6 \%$ & $18.6 \%$ & 0.0208 \\
\hline PCPS use & $2.4 \%$ & $3.5 \%$ & 0.06 \\
\hline \multicolumn{4}{|l|}{ Procedure } \\
\hline stent use & $90.1 \%$ & $91.0 \%$ & 0.42 \\
\hline DES use & $66.5 \%$ & $60.3 \%$ & 0.0016 \\
\hline \multicolumn{4}{|l|}{ Door to balloon time, minutes } \\
\hline Overall & $75(52-126)$ & $74(52-113)$ & 0.34 \\
\hline NSTEMI & $137(87-272)$ & $136(88-319)$ & 0.33 \\
\hline STEMI & $64(48-92)$ & $67(50-93)$ & 0.33 \\
\hline \multicolumn{4}{|l|}{ Final TIMI 3 flow } \\
\hline Overall & $92.7 \%$ & $91.0 \%$ & 0.11 \\
\hline NSTEMI & $95.0 \%$ & $93.0 \%$ & 0.41 \\
\hline STEMI & $91.7 \%$ & $90.4 \%$ & 0.32 \\
\hline Systolic blood pressure, $\mathrm{mmHg}$ & $139(118-159)$ & $138(117-161)$ & 0.43 \\
\hline Heart rate, bpm & $77(65-90)$ & $77(65-90)$ & 0.85 \\
\hline Low-density lipoprotein cholesterol, mg/dL & $115(92-140)$ & $114(88-142)$ & 0.32 \\
\hline High-density lipoprotein cholesterol, mg/dL & $46(39-54)$ & $44(37-53)$ & 0.0013 \\
\hline Triglyceride, $\mathrm{mg} / \mathrm{dL}$ & $95(64-148)$ & $97(59-157)$ & 0.91 \\
\hline Max CK, IU/L & $1232(410-2888)$ & $1691(627-3493)$ & $<0.0001$ \\
\hline \multicolumn{4}{|l|}{ Medication at discharge } \\
\hline Aspirin & $95.5 \%$ & $95.9 \%$ & 0.65 \\
\hline DAPT & $74.4 \%$ & $76.3 \%$ & 0.24 \\
\hline $\mathrm{OAC}$ & $12.1 \%$ & $13.1 \%$ & 0.42 \\
\hline ACEIs & $53.0 \%$ & $51.7 \%$ & 0.48 \\
\hline ARBs & $27.3 \%$ & $29.5 \%$ & 0.18 \\
\hline Beta blockers & $67.2 \%$ & $69.5 \%$ & 0.20 \\
\hline Statins & $86.4 \%$ & $87.4 \%$ & 0.42 \\
\hline
\end{tabular}

ACEI indicates angiotensin-converting enzyme; ARB, angiotensin-receptor blocker; CABG, coronary artery bypass graft surgery; CK, creatinine kinase; CKD, chronic kidney disease; DAPT, dual antiplatelets; IABP, intra-aortic balloon pumping; LAD, left anterior descending coronary artery; NSTEMI, non-STEMI; OAC, oral anticoagulant; PCI, percutaneous coronary intervention; PCPS, percutaneous cardiopulmonary support; STEMI, ST-segment elevation myocardial infarction; and TIMI, thrombolysis in myocardial infarction. 


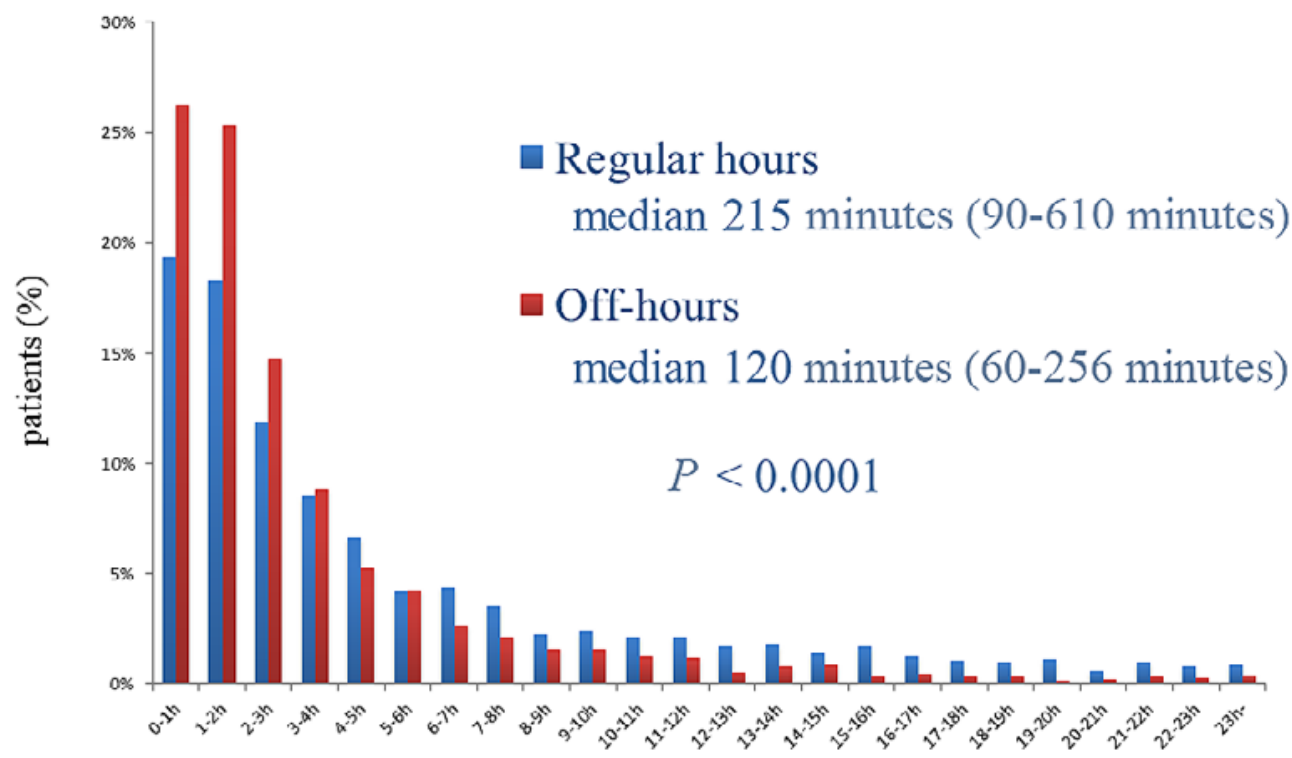

Time (hours)

Figure 2. Distribution of symptom onset-to-balloon time.

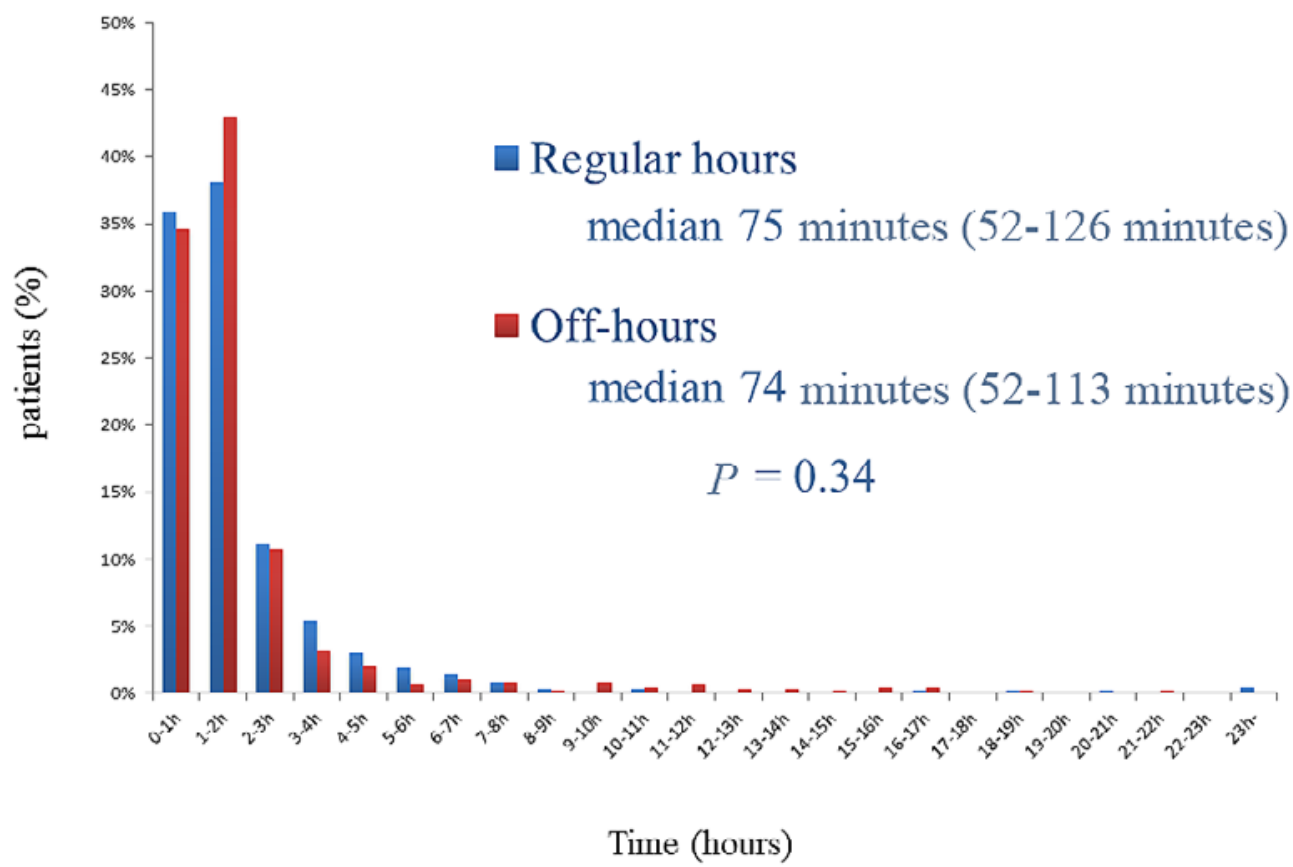

Figure 3. Distribution of door-to-balloon time.

system to avoid diagnostic and treatment delay might be necessary to further improve the outcomes of patients with AMI.

Limitations: This study is based on a post hoc analysis and thus is subject to potential influences that might have affected the results. Although the sample size was moderate, it might not have had sufficient statistical power to determine differences in outcome measurements between regular hours and off-hours. This was not a population- based study. The participating institutions are regional centers capable of advanced medical management. Therefore, not all patients who had AMI in the region during the study period were presented. In this study, the time from onset to presentation was significantly different between groups. The delay in presentation and prehospital management are associated with multiple factors including patient background, transfer system, ischemic preconditioning, and geographic distribution of quality of health- 

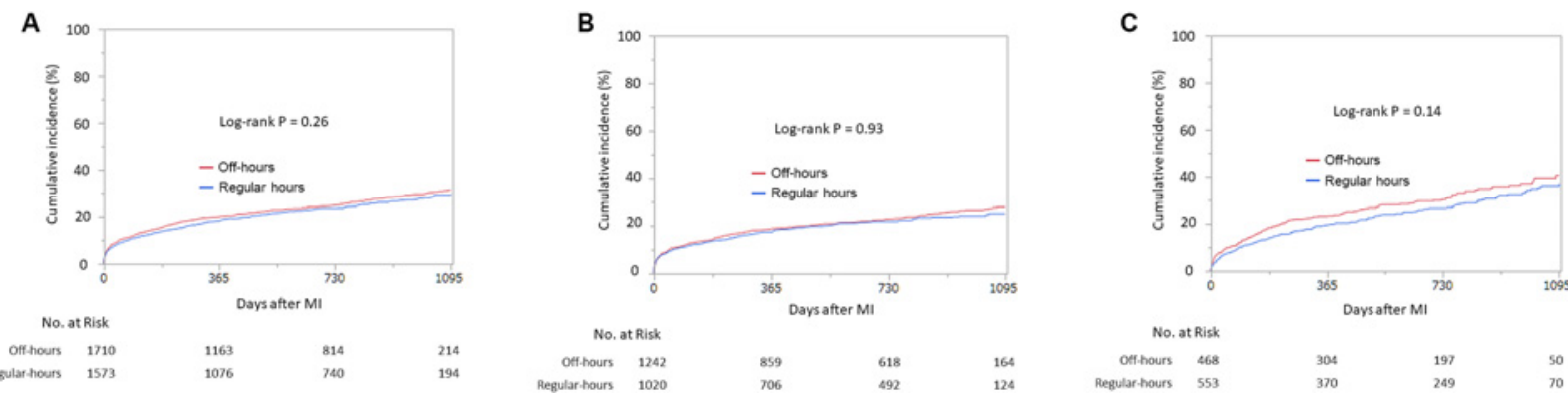

Figure 4. Kaplan-Meier curves of primary endpoints. A: Overall, B: STEMI, and C: NSTEMI.
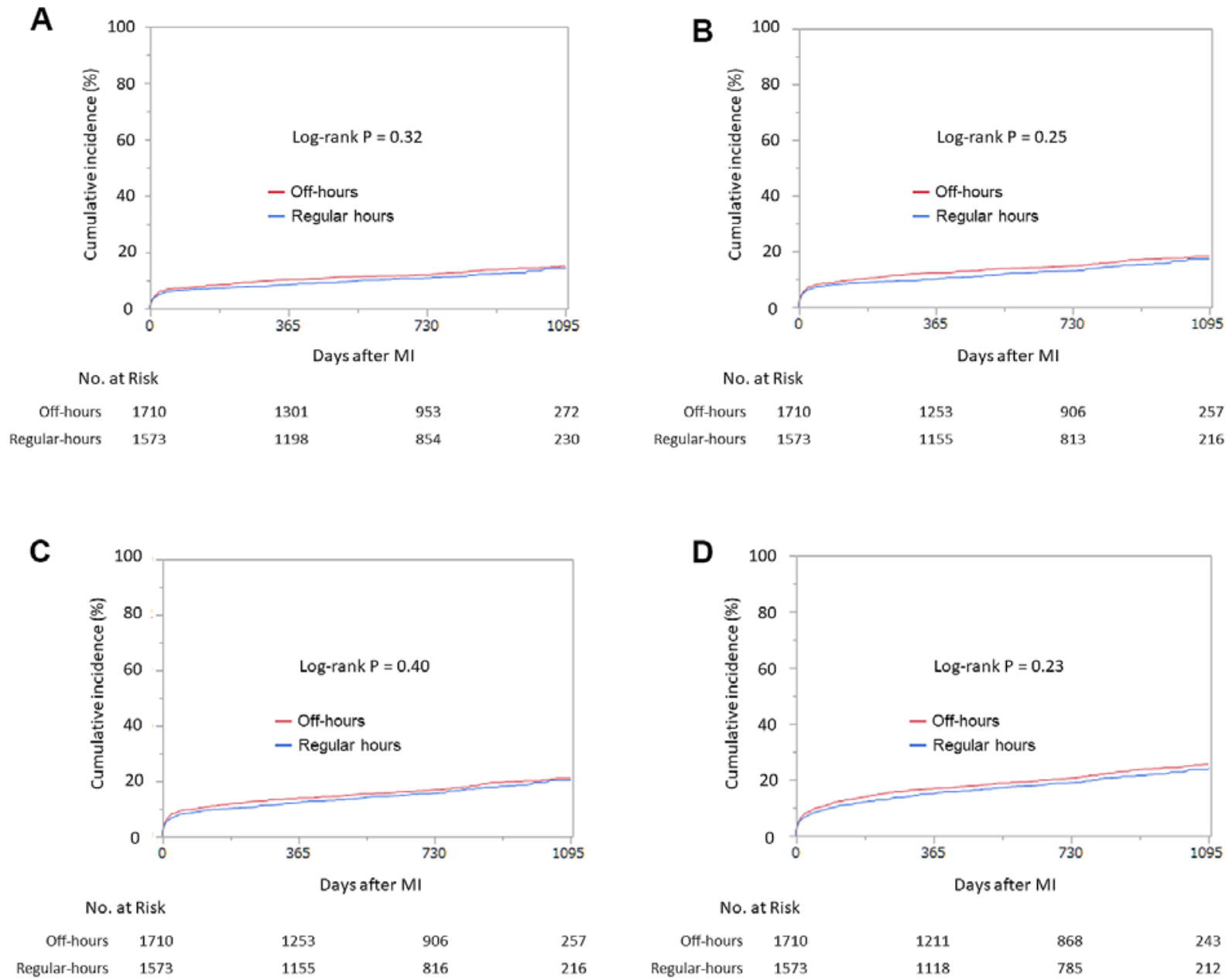

Figure 5. Kaplan-Meier curves of secondary endpoints. A: Death, B: composite of death and non-fatal MI, C: death, non-fatal MI and non-fatal stroke, and D: composite of death, non-fatal MI, non-fatal stroke, and cardiac failure.

care, and it is difficult to identify the prognostic factors derived from uncontrolled homogeneous observational studies. Finally, this study aimed to determine differences between presentation at hospitals during regular hours and off-hours. However, the most challenging cases in patients with AMI did not undergo triage and might not have been correctly transferred during off-hours, which might have created a selection bias for enrollment.

\section{Conclusions}

The clinical impact of presentation during regular hours or off-hours on the long-term clinical outcomes of patients with AMI in Japan is comparable in contemporary practice. 
Table II. Univariate and Multivariate Cox Regression Analysis for Primary Endpoint

\begin{tabular}{lcccccc}
\hline & \multicolumn{3}{c}{ Univariate } & \multicolumn{4}{c}{ Multivariate } \\
& HR & $95 \%$ CI & $P$ & HR & $95 \%$ CI & $P$ \\
\hline Versus regular-hour off-hour presentation & & & & & & \\
Overall & 1.08 & $0.80-1.06$ & 0.26 & 1.03 & $0.87-1.21$ & 0.26 \\
STEMI & 1.08 & $0.90-1.29$ & 0.40 & 1.01 & $0.82-1.23$ & 0.93 \\
NSTEMI & 1.19 & $0.95-1.49$ & 0.14 & 1.10 & $0.84-1.44$ & 0.47 \\
\hline
\end{tabular}

HR indicates hazard ratio; and 95\%CI, 95\% confidential interval. Other abbreviations as Table I. Multivariate model adjusted for age, sex, hypertension, diabetes, dyslipidemia, chronic kidney disease, current smoking, prior MI, prior stroke, Killip Class, type of MI, logarithm of time from onset to admission, urgent $\mathrm{CAG}$, primary $\mathrm{PCI}$ and urgent $\mathrm{CABG}$ (according to the primary paper of J-MINUET).

\section{Acknowledgments}

The authors thank all the enrolled patients, participating cardiologists, medical and other staff who contributed to this study. The J-MINUET investigators are listed in Appendix.

\section{Disclosure}

Conflicts of interest: The authors declare that there is no conflict of interest.

Trial registration: UMIN Unique trial Number: UMIN 000010037

\section{Appendix}

J-MINUET Investigators: Masaharu Ishihara, Hyogo College of Medicine (Chairperson); Hisao Ogawa, National Cerebral and Cardiovascular Center; Nobuaki Kokubu, Sapporo Medical University; Tadaya Sato, Akita Medical Center; Teruo Inoue, Dokkyo Medical University; Shigeru Oshima, Gunma Prefectural Cardiovascular Center; Hiroshi Funayama, Saitama Medical Center Jichi Medical University; Ken Kozuma, Hiroyuki Kyono, Teikyo University; Wataru Shimizu, Nippon Medical School; Satoru Suwa, Juntendo University Shizuoka Hospital; Kengo Tanabe, Mitsui Memorial Hospital; Tetsuya Tobaru, Sakakibara Heart Institute; Kazuo Kimura, Yokohama City University Medical Center; Junya Ako, Kitasato University; Mafumi Owa, Suwa Red Cross Hospital; Takahito Sone, Yasuhiro Sone, Ogaki Municipal Hospital; Yukio Ozaki, Fujita Health University; Satoshi Yasuda, Teruo Noguchi, Masashi Fujino, Yoshihiro Miyamoto, Kunihiko Nishimura, National Cerebral and Cardiovascular Center; Junichi Kotani, Osaka University Graduate School of Medicine; Takashi Morita, Osaka General Medical Center; Atsunori Okamura, Sakurabashi Watanabe Hospital; Yoshihiko Saito, Hiroyuki Okura, Nara Medical University; Masaaki Uematsu, Kansai Rosai Hospital; Shirou Uemura, Kawasaki Medical School; Atsushi Hirohata, The Sakakibara Heart Institute of Okayama; Yasuharu Nakama, Hiroshima City Hospital; Keijiro Saku, Fukuoka University School of Medicine; Seiji Hokimoto, Kumamoto University Graduate School of Medical Sciences; Koichi Nakao, Saiseikai Kumamoto Hospital; Kazuteru Fujimoto, National Hospital Organization Kumamoto Medical Center; Yoshisato Shibata, Miyazaki Medical Association Hospital; Kazuhito Hirata, Okinawa Prefectural Chubu Hospital.

\section{References}

1. Lozano R, Naghavi M, Foreman K, et al. Global and regional mortality from 235 causes of death for 20 age groups in 1990 and 2010: a systematic analysis for the Global Burden of Disease Study 2010. Lancet 2012; 380: 2095-128.

2. Authors/Task Force members, Windecker S, Kolh P, Alfonso F, et al. ESC/EACTS Guidelines on myocardial revascularization: the Task Force on Myocardial Revascularization of the European Society of Cardiology (ESC) ant the European Association for Cardio-Thoracic Surgery (EACTS) Developed with the special contribution of the European Association of Percutaneous Cardiovascular Intervention (EAPCI). Eur Heart J 2014; 35: 2541-619.

3. Thiele $\mathrm{H}$, Zeymer $\mathrm{U}$, Thelemann $\mathrm{N}$, et al. Intraaortic balloon pump in cardiogenic shock complicating acute myocardial infarction: long-term 6-year outcome of the randomized IABPSHOCK II trial. Circulation 2018; 139: 395-403.

4. Yamamoto K, Sakakura K, Akashi N, et al. Comparison of clinical outcomes between the ostial versus non-ostial culprit in proximal left anterior descending artery acute myocardial infarction. Int Heart J 2019; 60: 37-44.

5. De Luca G, Suryapranata H, Ottervanger JP, et al. Circadian variation in myocardial perfusion and mortality in patients with ST-segment elevation myocardial infarction treated by primary angioplasty. Am Heart J 2005; 150: 1185-9.

6. Glaser R, Naidu SS, Selzer F, et al. Factors associated with poorer prognosis for patients undergoing primary percutaneous coronary intervention during off-hours: biology or systems failure? JACC Cardiovasc Interv 2008; 1: 681-8.

7. Magid DJ, Wang Y, Herrin J, et al. Relationship between time of day, day of week, timeliness of reperfusion, and in-hospital mortality for patients with acute ST-segment elevation myocardial infarction. JAMA 2005; 294: 803-12.

8. Sorita A, Ahmed A, Starr SR, et al. Off-hour presentation and outcomes in patients with acute myocardial infarction: systematic review and meta-analysis. BMJ 2014; 348: f7393.

9. Sadeghi HM, Grines CL, Chandra HR, et al. Magnitude and impact of treatment delays on weeknights and weekends in patients undergoing primary angioplasty for acute myocardial infarction (the CADILLAC Trial). Am J Cardiol 2004; 94: 63740, A9.

10. Ortolani P, Marzocchi A, Marrozzini C, et al. Clinical comparison of "normal-hours" vs "off-hours" percutaneous coronary interventions for ST-elevation myocardial infarction. Am Heart J 2007; 154: 366-72.

11. Jneid H, Fonarow GC, Cannon CP, et al. Impact of time of presentation on the care and outcomes of acute myocardial infarction. Circulation 2008; 117: 2502-9.

12. Sorita A, Lennon RJ, Haydour Q, et al. Off-hour admission and outcomes for patients with acute myocardial infarction undergoing percutaneous coronary interventions. Am Heart J 2015; 169: $62-8$. 
13. Bell CM, Redelmeier DA. Mortality among patients admitted to hospitals on weekends as compared with weekdays. N Engl J Med 2001; 345: 663-8.

14. Casella G, Ottani F, Ortolani P, et al. Off-hour primary percutaneous coronary angioplasty does not affect outcome of patients with ST-segment elevation acute myocardial infarction treated within a regional network for reperfusion: the REAL (Registro Regionale Angioplastiche dell'Emilia-Romagna) registry. JACC Cardiovasc Interv 2011; 4: 270-8.

15. Ogita M, Suwa S, Ebina H, et al. Off-hours presentation does not affect in-hospital mortality of Japanese patients with acute myocardial infarction: J-MINUET substudy. Journal of Cardiology 2017; 70: 553-8.

16. Ishihara $\mathrm{M}$, Fujino $\mathrm{M}$, Ogawa $\mathrm{H}$, et al. Clinical presentation, management and outcome of Japanese patients with acute myocardial infarction in the troponin era - Japanese registry of acute myocardial infarction diagnosed by universal definition (JMINUET). Circ J 2015; 79: 1255-62.

17. Thygesen K, Alpert JS, Jaffe AS, et al; Joint ESC/ACCF/AHA/ WHF Task force. Third universal definition of myocardial infarction. J Am Coll Cardiol 2012; 60: 1581-98.

18. Ishihara M, Nakao K, Ozaki Y, et al; on behalf of J-MINUET Investigators. Long-term outcomes of non-ST-elevation myocardial infarction without creatine kinase elevation - The JMINUET Study. Circ J 2017; 81: 958-65.

19. Ryan JW, Peterson ED, Chen AY, et al. Optimal timing of intervention in non-ST-segment elevation acute coronary syndromes: insights from the CRUSADE (Can Rapid risk stratification of Unstable angina patients Suppress ADverse outcomes with Early implementation of the ACC/AHA guidelines) Registry. Circulation 2005; 112: 3049-57.

20. Redelmeier DA, Bell CM. Weekend worriers. N Engl J Med 2007; 356: 1164-5.

21. Shah AP, French WJ. Physicians... wake up! JACC Cardiovasc Interv 2008; 1: 689-91.

22. Berger A, Meier JM, Wasserfallen JB, et al. Out of hours percutaneous coronary interventions in acute coronary syndromes: long-term outcome. Heart 2006; 92: 1157-8.

23. Kostis WJ, Demissie K, Marcella SW, et al. Weekend versus weekday admission and mortality from myocardial infarction. $\mathrm{N}$ Engl J Med 2007; 356: 1099-109.

24. de Boer SP, Oemrawsingh RM, Lenzen MJ, et al. Primary PCI during off-hours is not related to increased mortality. Eur Heart J Acute Cardiovasc Care 2012; 1: 33-9.
25. Redfors B, Dworeck C, Angerås O, et al. Prognosis is similar for patients who undergo primary PCI during regular-hours and off-hours: A report from SCAAR. Catheter Cardiovasc Interv 2018; 91: 1240-9.

26. Cubeddu RJ, Palacios IF, Blankenship JC, et al. Outcome of patients with ST-segment elevation myocardial infarction undergoing primary percutaneous coronary intervention during on- versus off-hours (A Harmonizing Outcomes with revascularization and Stents in acute myocardial infarction [HORIZONS-AMI] trial substudy). Am J Cardiol 2013; 111: 946-54.

27. Angeja BG, Gibson CM, Chin R, et al. Participants in the National Registry of Myocardial Infarction 2-3. Predictors of doorto-balloon delay in primary angioplasty. Am J Cardiol 2002; 89: $1156-61$.

28. Blankenship JC, Skelding KA, Scott TD, et al. Predictors of reperfusion delay in patients with acute myocardial infarction undergoing primary percutaneous coronary intervention from the HORIZONS-AMI trial. Am J Cardiol 2010; 106: 1527-33.

29. Scholz KH, Maier SKG, Maier LS, et al. Impact of treatment delay on mortality in ST-segment elevation myocardial infarction (STEMI) patients presenting with and without haemodynamic instability: results from the German prospective, multicentre FITT-STEMI trial. Eur Heart J 2018; 39: 1065-74.

30. Dasari TW, Roe MT, Chen AY, et al. Impact of time of presentation on process performance and outcomes in ST-segmentelevation myocardial infarction: a report from the American Heart Association: Mission Lifeline program. Circ Cardiovasc Qual Outcomes 2014; 7: 656-63.

31. Hannan EL, Zhong Y, Jacobs AK, et al. Effect of onset-to-door time and door-to-balloon time on mortality in patients undergoing percutaneous coronary interventions for ST-segment elevation myocardial infarction. Am J Cardiol 2010; 106: 143-7.

32. Shiomi H, Nakagawa Y, Morimoto T, et al. Association of onset to balloon and door-to-balloon time with long term clinical outcome in patients with ST elevation acute myocardial infarction having primary percutaneous coronary intervention: observational study. BMJ 2012; 344: e3257.

33. Ikegami $\mathrm{N}$, Yoo BK, Hashimoto $\mathrm{H}$, et al. Japanese universal health coverage: evolution, achievements, and challenges. Lancet 2011; 378: 1106-15.

34. Nakatsuma K, Shiomi H, Morimoto T, et al. Inter-facility transfer vs. direct admission of patients with ST-segment elevation acute myocardial infarction undergoing primary percutaneous coronary intervention. Circ J 2016; 80: 1764-72. 